\title{
Two Successive Type II Radio Bursts Associated With B-Class Flares and Slow CMEs
}

\author{
Suli $\mathrm{Ma}^{1 *}$ and Huadong Chen ${ }^{1,2}$ \\ ${ }^{1}$ CAS Key Laboratory of Solar Activity, National Astronomical Observatories, Chinese Academy of Sciences, Beijing, China, \\ ${ }^{2}$ School of Astronomy and Space Science, University of Chinese Academy of Sciences, Beijing, China
}

OPEN ACCESS

Edited by:

Dipankar Banerjee,

Indian Institute of Astrophysics, India

Reviewed by:

Mingde Ding,

Nanjing University, China

Debi Prasad Choudhary,

California State University, Northridge

United States

*Correspondence:

Suli Ma

sma@nao.cas.cn

Specialty section:

This article was submitted to

Stellar and Solar Physics,

a section of the journal

Frontiers in Astronomy and Space

Sciences

Received: 03 June 2019 Accepted: 07 April 2020

Published: 12 May 2020

Citation:

Ma S and Chen H (2020) Two

Successive Type II Radio Bursts Associated With B-Class Flares and

Slow CMEs.

Front. Astron. Space Sci. 7:17.

doi: 10.3389/fspas.2020.00017
From 2018 Oct 12 to 13, three successive solar eruptions (E1-E3) with B-class flares and poor white light coronal mass ejections (CMEs) occurred from the same active region NOAA AR 12724. Interestingly, the first two eruptions are associated with Type II radio bursts but the third is not. Using the soft X-ray flux data, radio dynamic spectra and dual perspective EUV intensity images, we comparatively investigate the three events. Our results show that their relevant flares are weak (B2.1, B7.9, and B2.3) and short-lived (13, 9, and $14 \mathrm{~min})$. The main eruption directions of E1 and E2 are along $\sim 45^{\circ}$ north of their radial directions, while E3 primarily propagated along the radial direction. In the EUV channels, the early speeds of the first two CMEs have apparent speeds of $\sim 320$ and $\sim 380 \mathrm{~km} \mathrm{~s}^{-1}$, which could exceed their respective local Alfvén speeds of $\sim 300$ and $\sim 350 \mathrm{~km} \mathrm{~s}^{-1}$. However, the CME in the third eruption possesses a much lower speed of $\sim 160 \mathrm{~km} \mathrm{~s}^{-1}$. These results suggest that the observed Type II radio bursts in the eruptions E1 and E2 are likely triggered by their associated CMEs and the direction of eruption and the ambient plasma and magnetic environments may take an important place in producing Type II radio burst or shock as well.

Keywords: radio radiation, coronal mass ejections (CMEs), UV radiation, flares, corona, filaments, shock waves, magnetic topology

\section{INTRODUCTION}

Solar Type II radio bursts were first reported by Payne-Scott et al. (1947) and named by Wild and McCready (1950). Usually, a Type II burst appeared as a slowly drifting, from high to low frequencies, narrow frequency band patterns in radio dynamic spectra (e.g., Nelson and Melrose, 1985). It is generally believed that type II bursts are excited by magnetohydrodynamics (MHD) shocks in the solar atmosphere (e.g., Nelson and Melrose, 1985; Cliver et al., 1999; Nindos et al., 2008, 2011; Vršnak and Cliver, 2008, and references therein).

Since the first discovery of Type II bursts, they have been found to be closely related to both flares (e.g., Wild et al., 1954; Maxwell and Thompson, 1962; Dodge, 1975) and high-velocity ejections (e.g., Dodson et al., 1953; Giovanelli and Roberts, 1958; Swarup et al., 1960). The blast wave initiated by flares and piston-driven mechanism associated with coronal mass ejections (CMEs) have become the two main competitors of the triggering mechanism of the Type II bursts as reviewed by the papers (e.g., Cliver et al., 1999; Vršnak and Cliver, 2008; Nindos et al., 2011) and also suggested by some recent studies (e.g., Zheng et al., 2018; Eselevich et al., 2019; Frassati et al., 2019). In the piston-driven mechanism, besides CME front, some other triggers, such as soft $\mathrm{X}$-ray jet, erupting coronal loop and eruptive magnetic flux rope are also proposed to explain the 
production of shock or Type II burst (e.g., Klein et al., 1999; Klassen et al., 2003; Dauphin et al., 2006; Su et al., 2015; Eselevich et al., 2017; Grechnev et al., 2018).

In theory, the occurrence of a coronal shock requires the introduction of a sudden disturbance in the corona, which should travel with a speed faster than the local Alfvén velocity. Early studies (e.g., Gosling et al., 1976) indicate that the velocities of CMEs with type II bursts would exceed $400-550 \mathrm{~km} \mathrm{~s}^{-1}$. However, Gopalswamy et al. (2001) made a statistical study and found that $50 \%$ of limb CMEs associated Type II bursts during 1995-1997 have speeds lower than $500 \mathrm{~km} \mathrm{~s}^{-1}$ and the lower cutoff of these CMEs' speeds may reach $\sim 250 \mathrm{~km} \mathrm{~s}^{-1}$. So far, detailed case studies about Type II radio bursts with slow CMEs and weak flares (below C-class) have been very rare.

In this paper, we present a case study about two successive Type II bursts associated with B-class flares and CMEs with slow speeds below $\sim 400 \mathrm{~km} \mathrm{~s}^{-1}$ in the period of solar activity minimum. Our comparative investigations suggest that these Type II radio bursts are likely triggered by their associated CMEs and we also discuss the influence from the ambient coronal magnetic structures on the eruptions.

\section{OBSERVATIONS}

From 2018 Oct 12 to 13 , three eruptions orderly took place in the active region (AR) NOAA 12724. AR 12724 is near the solar east limb in the field of view (FOV) of the ground-based telescopes (spectrographs) or space-based telescopes on geosynchronous satellites, such as the Solar and Heliospheric Observatory SOHO and the Solar Dynamics Observatory (SDO, Pesnell et al., 2012). The Solar Terrestrial Relations Observatory (STEREO, Kaiser et al., 2008) consists of two space-based observatories-one ahead of Earth in its orbit (STA), the other trailing behind (STB, lost communications since 2014 Oct 1). The STA orbits the Sun with a radius slightly smaller than $1 \mathrm{AU}$ and the separation angle between STA and the Earth was about $105^{\circ}$ during the three events. The host AR is located on the disk in the view of STA.

We use the dynamic spectrum data from the radio spectrograph ORFEES (Observation Radio Frequence pour l'Etude des Eruptions Solaires) observing between 140 and 1,000 $\mathrm{MHz}$, Learmonth solar radio spectrograph covering a frequency range of 25-180 MHz (LEAR, Kennewell and Steward, 2003), and the CALLISTO spectrometer (Benz et al., 2009) at the Greenland Observatory. Intensity images provided by the Atmospheric Imaging Assembly (AIA, Lemen et al., 2012) on SDO and the EUV Imager (EUVI, Wuelser et al., 2004) in the Sun Earth Connection Coronal and Heliospheric Investigation (SECHHI; Howard et al., 2008) on STA are also utilized to study the early stages of the eruptions. The observation from the Large Angle and Spectrometric Coronagraph (LASCO; Brueckner et al., 1995) on-board SOHO with a FOV of 2-6.0 $\mathrm{R}_{\odot}$ help us to check the associated CMEs.

\section{RESULTS}

\subsection{Overview of the Events}

The general information of the three successive eruption events (E1-E3) are listed in Table 1. All the three events originated from the same active region (AR) NOAA 12724 and each of them involved a filament eruption, a B-class flare and a slow poor white light (WL) CME. Type II radio bursts only appeared in E1 and E2. The detailed magnitude and the start, peak, and end time of each flare are given in the "Flare Class" and "Flare Time" column, respectively. The central locations of the relevant filaments $(\mathrm{F} 1-\mathrm{F} 3)$ in the FOV of AIA are presented in the "Filament Center" column. The CMEs' angular widths (AWs) and their quadratic speeds (obtained by performing second-order polynomial fittings to the height-time measurements) at the final height measurements are placed in the "CME AW" and "CME Speed" columns, respectively.

\subsection{Filaments}

The AIA $304 \AA$ (left column) and EUVI A $195 \AA$ (right column) images in Figure 1 show the morphologies of the AR 12724, the erupting filaments $(\mathrm{F} 1-\mathrm{F} 3)$ and their corresponding flares (Flare1-Flare3). In the $304 \AA$ images, it can be seen that F1 and F2 almost have a north-south orientation, while F3 runs from east to west. According to the EUVI A $195 \AA$ observations, Flare 1 and Flare 2 were located in the northwest of AR 12724, while Flare 3 mainly lay in the AR's southeast. To the east of AR 12724, a small emerging active region labeled "New" appeared to have nothing to do with the eruptions.

\subsection{Flares and Radio Bursts}

The GOES X-ray fluxes and radio dynamic spectrums associated with E1, E2 and E3 are shown in the panels (a) and (b) of Figures 2-4, respectively. Figure 2a displays the short-lived B2.1 Flare1. In Figure 2b, a type II radio burst can be found in the Learmonth's radio dynamic spectrum, suggesting that a shock (Shock1) was generated during E1. The type II radio burst, including a fundamental band $(\mathrm{F})$ and a harmonic band $(\mathrm{H})$ with two splitting lanes $\left(\mathrm{H}_{L}\right.$ and $\left.\mathrm{H}_{U}\right)$, started from $\sim 01: 52 \mathrm{UT}$, when Flare 1 has entered its descending phase. After $\sim 02: 00$ UT, it gradually disappeared. The frequencies along $\mathrm{F}, \mathrm{H}_{L}$ and $\mathrm{H}_{U}$ change from about 40,78 , and $80 \mathrm{MHz}$ to 26,54 , and 64 $\mathrm{MHz}$, respectively. Their average frequency drift rates are -0.086 , -0.087 , and $-0.109 \mathrm{MHz} \mathrm{s}^{-1}$. We adopt the frequency values represented by the black $(\mathrm{F})$, red $\left(\mathrm{H}_{L}\right)$, and blue $\left(\mathrm{H}_{U}\right)$ dashed curves to measure local plasma densities and further derive the speeds of Shock1 (see section 3.6).

It is generally believed that the band-splitting is caused by the emission from the upstream and downstream shock regions and the downstream/upstream density jump $(X)$ could provide an estimate of the coronal Alfvén speed (e.g, Smerd et al., 1974; Mann et al., 1995; Vršnak, 2001). The density jump can be described as

$$
X=\frac{n_{2}}{n_{1}}=\left(\frac{f_{U}}{f_{L}}\right)^{2}
$$

(Vršnak et al., 2002). Here, $n_{1}$ and $n_{2}$ are the electron densities of the plasma at the frequency $f_{L}$ in the lower frequency branch and at the frequency $f_{U}$ in the upper frequency branch of the harmonic bands, respectively. For the Type II radio burst in E1, we take $f_{U}=77.5$ and $f_{L}=64.5$ at $01: 54: 32$ UT (indicated by the vertical line in Figure 2b) and obtain $X=1.44$, indicating that Shock1 is a weak shock. Under the quasi-perpendicular shock 
TABLE 1 | General information of the eruptions.

\begin{tabular}{|c|c|c|c|c|c|c|c|}
\hline \multirow[t]{2}{*}{ Event } & \multirow[t]{2}{*}{ Date } & Flare & Flare time (UT) (start peak end) & Radio & Filament & CME AW & CME Speed \\
\hline & & class & & burst & center & $\left({ }^{\circ}\right)$ & $(\mathrm{km} / \mathrm{s})$ \\
\hline E1 & 2018 Oct 12 & B2.1 & 01:43 01:50 01:56 & $\|$ & $-900^{\prime \prime},-150^{\prime \prime}$ & 46 & 333 \\
\hline E2 & 2018 Oct 12 & B7.1 & $14: 04$ 14:08 14:13 & II, IIIs, IV & $-850^{\prime \prime},-160^{\prime \prime}$ & 44 & 492 \\
\hline E3 & 2018 Oct 13 & B2.3 & 13:28 13:34 13:42 & III & $-800^{\prime \prime},-240^{\prime \prime}$ & 38 & 133 \\
\hline
\end{tabular}

approximation and a plasma beta $\beta->0$, the Alfvén Mach number $M_{A}$ is related to the compression $X$ as

$$
M_{A}=\sqrt{\frac{X(X+5)}{2(4-X)}}
$$

Then, $M_{A}$ around 1.35 can be derived for Shock1.

The GOES X-ray flux of the B7.1-class flare (Flare2) and the composite dynamic spectrum from ORFEES $(144-400 \mathrm{MHz})$ and Greenland (25-105 MHz) associated with E2 are displayed in the top and bottom panels of Figure 3, respectively. A variety of radio bursts, such as a group of type III bursts (IIIs), a relatively strong short-lived type IV burst and a weak type II burst, can be found in the composite dynamic spectrum. The Type III bursts occurred in the initial and impulsive phase of Flare2. Some of them show negative frequency drifts which probably result from energetic electron beams propagating outward along open coronal magnetic field (e.g., Yan et al., 2006; Huang et al., 2011), while others show positive drifts which may be caused by energetic electron beams propagating downward from where they are accelerated (likely the reconnection region) (e.g., Reid and Ratcliffe, 2014; Ning, 2016; Tan et al., 2016). The type IV burst appeared after the peak of Flare2 and lasted for about $2 \mathrm{~min}$. It is probably excited by the energetic electrons trapped within the erupting magnetic structures (e.g., Smerd and Dulk, 1971; Vlahos et al., 1982; Stewart, 1985).

At around 14:15 UT on Oct 12, the type II burst appeared with obvious fundamental (F) and second harmonic bands (splitting into two lanes $\mathrm{H}_{L}$ and $\mathrm{H}_{U}$ ) in the observation of Greenland. Similar to E1, it likely indicates a shock (Shock2) induced during the eruption E2. Along $\mathrm{H}_{L}$, the frequency varies from 90 to $74 \mathrm{MHz}$ with a mean frequency drift of $-0.099 \mathrm{MHz} \mathrm{s}^{-1}$ and that decreases from $98 \mathrm{MHz}$ to $83 \mathrm{MHz}$ with a mean frequency drift of $-0.113 \mathrm{MHz} \mathrm{s}^{-1}$ along $\mathrm{H}_{U}$. In addition, a very faint third harmonic band $(3 \mathrm{H})$ seems to appear in the dynamic spectrum of ORFEES. Using the same method described above (see Equation 1), we obtained the density jump of Shock2 at 14:15:54 UT (marked by the vertical line in Figure $3 \mathbf{b}$, when the $\mathrm{H}_{U}$ is $98 \mathrm{MHz}$ and $\mathrm{H}_{L}$ is $86 \mathrm{MHz}$ ), which is about 1.30. The corresponding shock Mach number was deduced as 1.23.

Figure 4 shows the GOES X-ray flux of the flare (top panel) and the dynamic spectra (bottom panel) during E3. A B2.3-class flare (Flare3) correlated with E3. Taking advantage of the joint observations from ORFEES and Greenland, we found that only a very weak type III burst and no sign of type II burst appeared in this event.

\subsection{White Light CMEs}

The running difference intensity images of LASCO C2 and AIA at $193 \AA$ are composited and shown in Figure 5, displaying the coronal changes during E1, E2, and E3. Three faint stream-like CMEs (CME1-CME3) can be separately identified in the three eruptions. They are indicated by the arrows in the left, middle and right column of Figure 5, respectively. According to the LASCO CME catalog (see https://cdaw.gsfc.nasa.gov/CME_list), CME1CME3 only appear in the FOV of $\mathrm{C} 2\left(2 \sim 6 \mathrm{R}_{\odot}\right)$ and belong to "poor CMEs." They separately have an angular width of about 46,44 , and $38^{\circ}$, and a 2 nd-order speed at the time of final height measurement of 333, 492, and $133 \mathrm{~km} \mathrm{~s}^{-1}$ (see Table 1). The times of their first appearance in the LASCO C2's FOV are around 02:48 UT (Oct 12), 15:12 UT (Oct 12), and 14:36 UT (Oct 13), respectively. Obviously, the Type II bursts in E1 and E2 had already formed before their corresponding CMEs came into the C2 FOV. No relevant CME can be found from the observations of COR 1 (inner coronagraph) and COR 2 (outer coronagraph) on-board STA. This may be related to the on-disk perspective of STA and the weak magnitudes of the eruptions.

\subsection{Eruptions in EUV}

The AIA $193 \AA$ (Figure 6) and EUVI $195 \AA$ (Figure 7) intensity images display the early evolutions of E1-E3 from different perspectives. The original images in the left columns of Figures 6, 7 are utilized as references to get the base difference images in the middle and right columns. The AIA $193 \AA$ A data clearly exhibit the dome-like structures of CME1 and CME2 with distinct leading edges (LE1 and LE2) in their early stages, while CME3 had a tenuous leading edge (LE3). Comparing the main eruption directions of E1-E3 (indicated by the yellow arrows in Figure 6) with the radial directions (denoted by the purple arrows in Figure 6), it can be found that CME1 and CME2 primarily propagated northeast, whereas CME3 was ejected approximately along the radial direction.

In the EUVI observations, three diffusing EUV waves (W1W3) can be observed, as indicated by the orange arrows in Figure 7. EUV waves are also called "EIT waves" or global coronal waves, which are large-amplitude waves initially driven by the rapid lateral expansion of a CME in the low corona and later propagating freely (cf. Long et al., 2017). Ma et al. (2009) showed the evidence that EUV wave front includes contribution from its associated CME at the early stage. More information about the "EUV waves" could be seen in the recent reviews (Liu and Ofman, 2014; Warmuth, 2015; Chen, 2016; Long et al., 2017). The propagation directions of $\mathrm{W} 1$ and $\mathrm{W} 2$ are similar and mainly toward the north from their eruption centers, while 


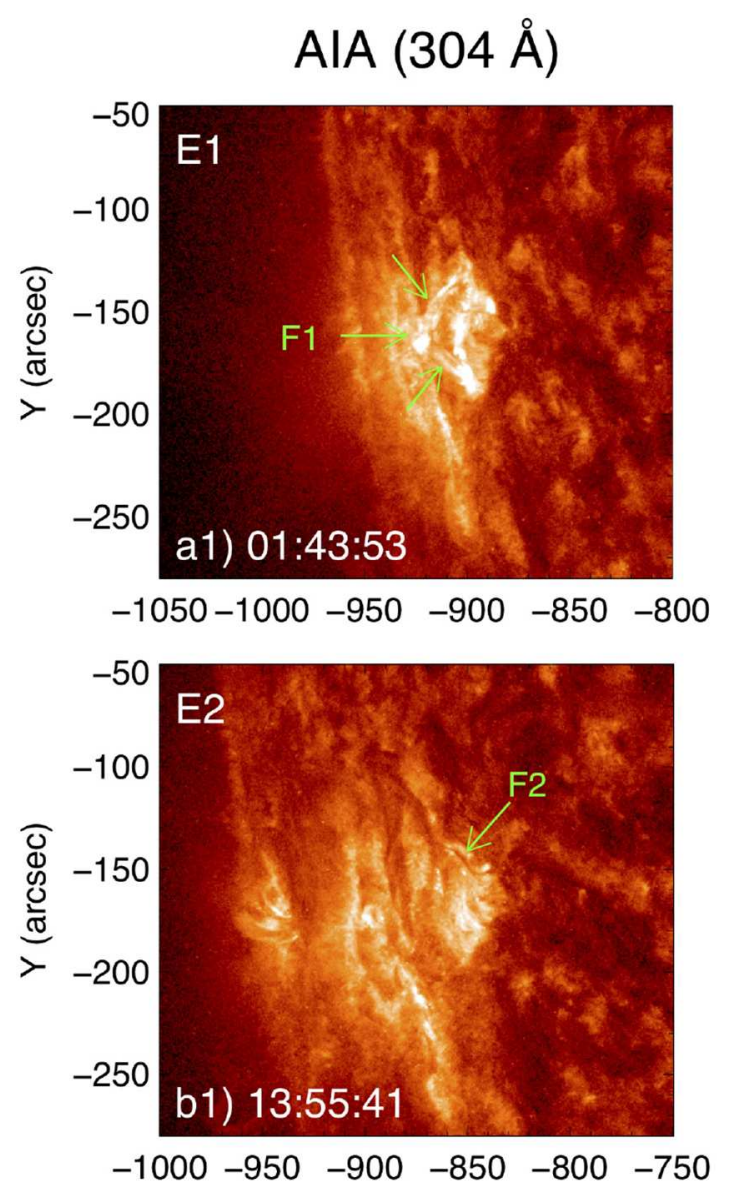

EUVI A (195 ^̊)
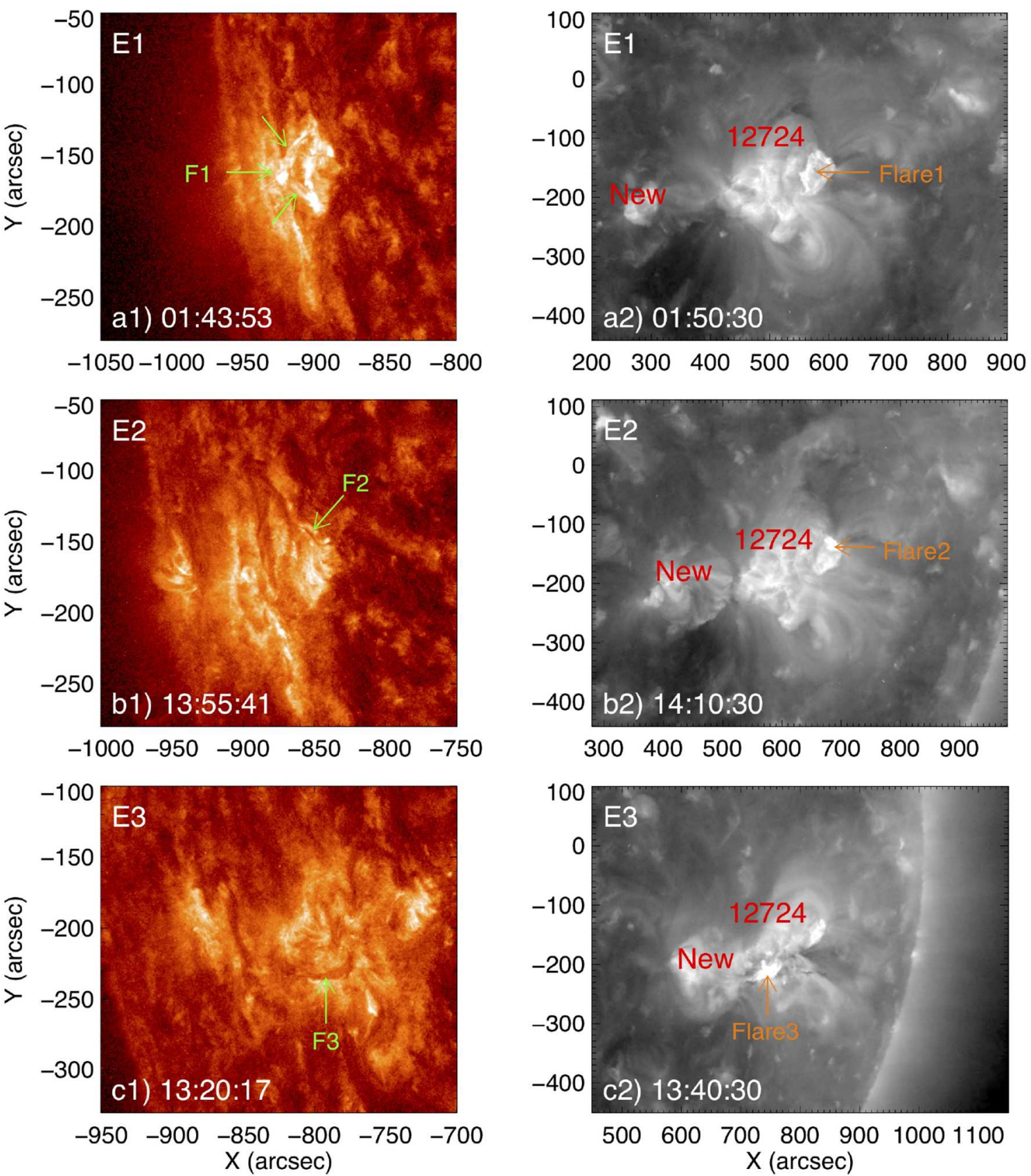

FIGURE 1 | Observations in EUV passbands displaying the morphology of the host active region NOAA 12724 during the occurrences of E1, E2, and E3. (a1,b1) and (c1) show the AIA observation at $304 \AA$ during E1, E2 and E3, respectively, and the green arrows point to the locations of filament1 (F1), filament2 (F2), and filament3 (F3). (a2,b2) and (c2) are EUVI images at $195 \AA$ during E1, E2 and E3, respectively, and the orange arrows direct the positions of Flare1, Flare2, and Flare3.

the traveling of W3 has no obvious preference. Combining the AIA and EUVI observations with a separation angle of $105^{\circ}$, we estimate that the eruption directions of E1 and E2 are alike along the $\sim 45^{\circ}$ north of their radial directions and E3 basically erupted radially.
Interestingly, a special brightening area ("SBA," Figures 6b2, 7b2) was observed at the north border of AR 12724 when the north flank of CME2 swept there (from $\sim 14: 09$ to $\sim 14: 11$ UT on Oct 12). It showed as an arc structure in the AIA images and a brow-like brightening in the EUVI images. The 

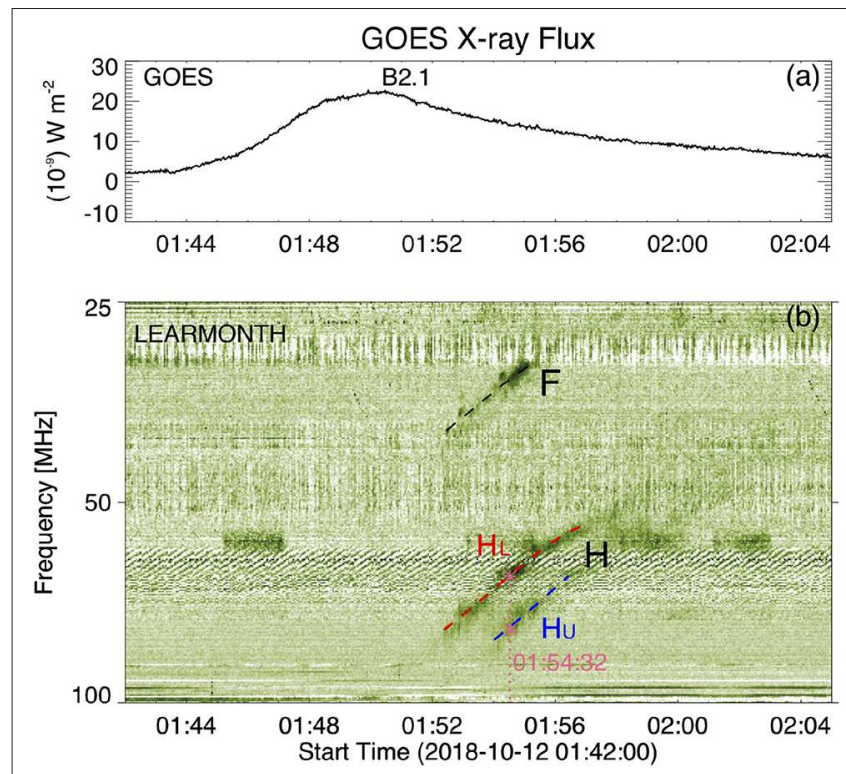

FIGURE 2 | GOES soft X-ray flux during Flare1 in 1-8 $\AA$ (a) and a radio dynamic spectrum from Learmonth showing the information about the associated Type II radio burst (b). "F" indicates the fundamental frequency band and " $H$ " mark the harmonic frequency band with the lower (" $H_{L}$ ") and higher (" $\mathrm{H}_{U}$ ") splitting branches. The three curves along the fundamental (black), lower (red), and higher (blue) harmonic splitting bands are used to measure the frequency drifts and the height and speed of Shock1. The vertical line in (b) indicate the time at 01:54:32 UT. The two pink asterisks mark the frequencies which are applied to calculate the density compression of Shock1.

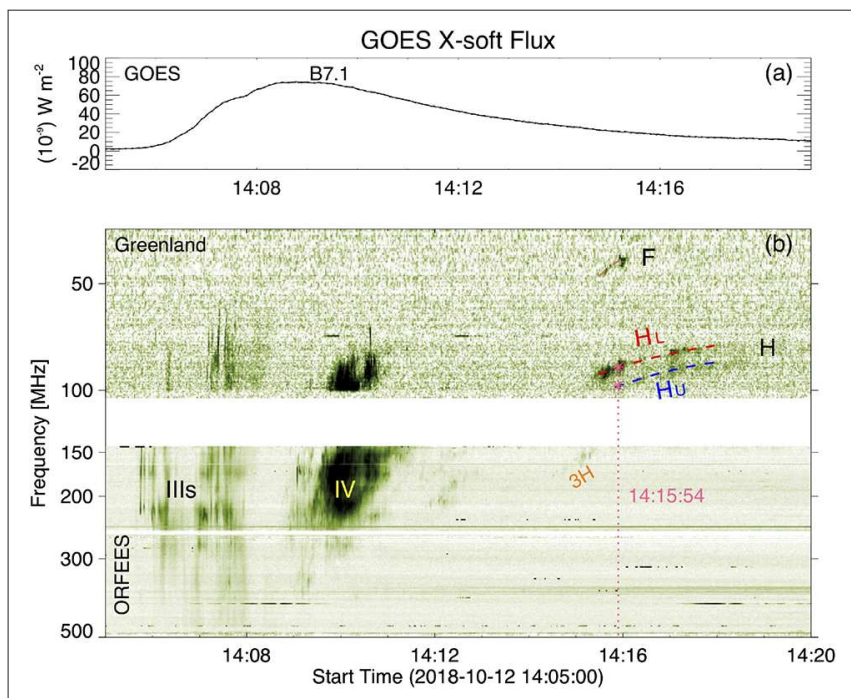

FIGURE 3 | GOES soft X-ray flux during Flare2 in 1-8 $\AA$ (a) and the composite radio dynamic spectrum from Greenland (25-105 $\mathrm{MHz}$ ) and ORFEES (144-400 MHz) displaying the complicated radio bursts during E2 (b). Along the red and blue dashed curves in (b) we will measure the frequency drift of the Type II radio burst and derive the height and speed of the associated shock (Shock2). The vertical line in (b) indicate the time at 14:15:54 UT. The two pink in (b) asterisks mark the frequencies which are used to calculate the density compressions of Shock2.

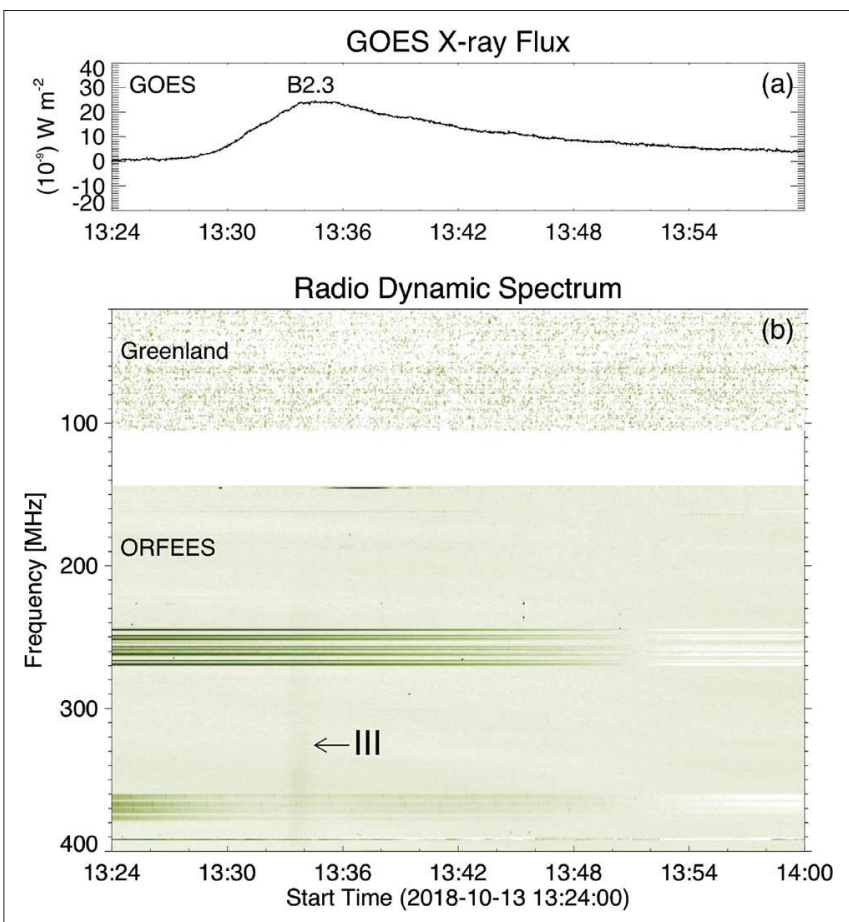

FIGURE 4 | GOES soft X-ray flux in 1-8 $\AA$ during Flare3 (a) and a composite radio dynamic spectrum from Greenland $(25-105 \mathrm{MHz})$ and ORFEES (144-400 MHz) during E3 (b).

SBA may be caused by the interaction of CME or EUV waves with some coronal structures. Later, another expanding domelike structure and a propagating diffusing wave front can be detected to propagate forward through SBA in the AIA and EUVI intensity images, which are indicated by the blue arrows with " $2 \mathrm{ND}$ ” in Figures 6b3,b4, 7b3, respectively. It is probably a secondary wave.

\subsection{Kinetics of the CMEs and Shocks}

In order to explore the relationship between the CMEs and their associated Type II radio bursts, we studied the kinetics of CME1CME3 and Shock1-Shock2, which are presented in Figure 8. For convenience, the identical colors represent the same bands or slits in Figures 2, 3, 6, 8. Because there is no associated radioheliograph observation to be available, the exact locations of Type II burst sources are hard to be determined. Assuming that the electron density of the corona varies with heliocentric distance and the shock propagates along the radial direction, we first deduced the local plasma densities $(n)$ of Shock1 and Shock2 from the observed frequency $\left(f_{p}\right.$ indicated by the black dashed line in Figure 2 or $2 f_{p}$ indicated by the red and blue dashed lines in Figures 2, 3) in the light of their relationship

$$
f_{p}=8.98 \times 10^{3} \sqrt{n}
$$




\section{E1}

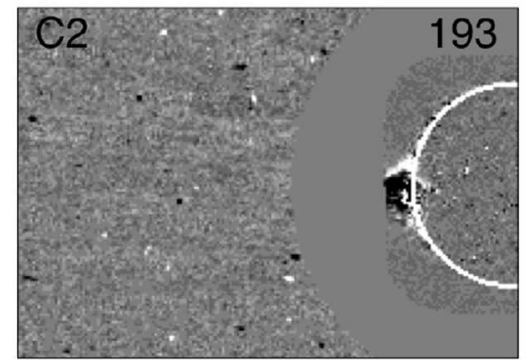

(a1) $2018101202: 00$

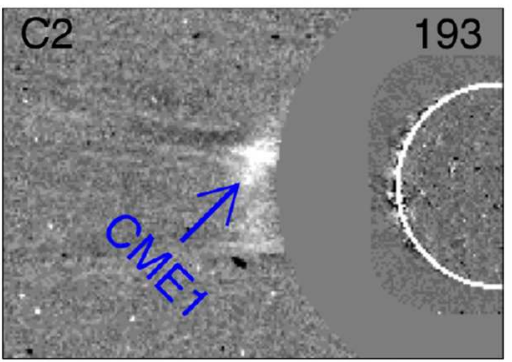

(a2) $2018101202: 48$

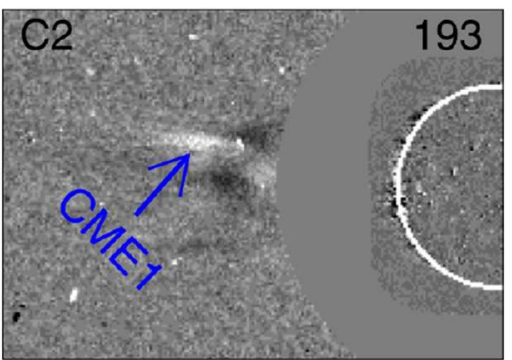

(a3) $2018101203: 24$
E2

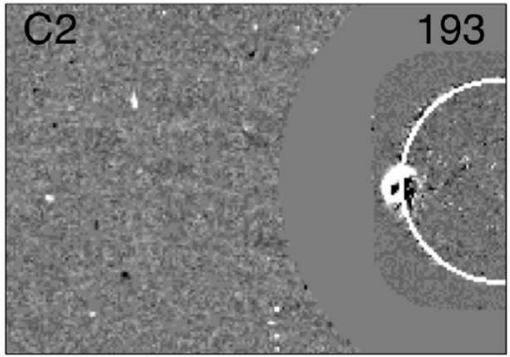

(b1) $2018101214: 12$

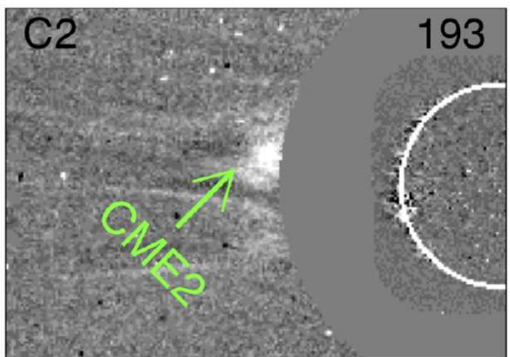

(b2) 20181012 15:12

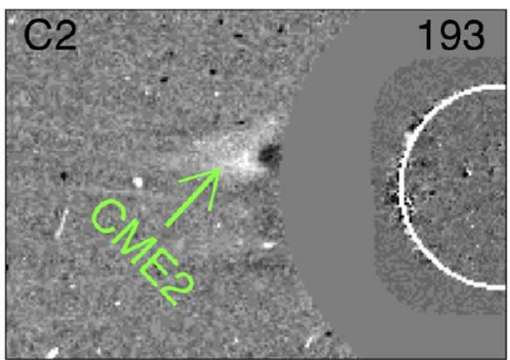

(b3) $2018101215: 24$
E3

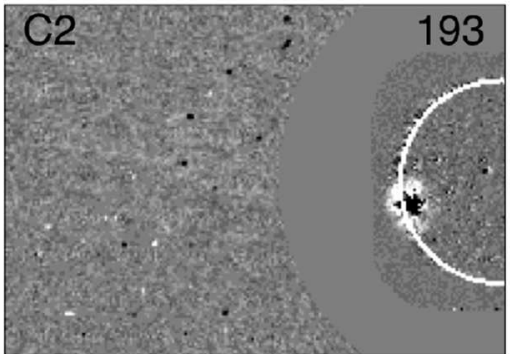

(c1) $2018101313: 36$

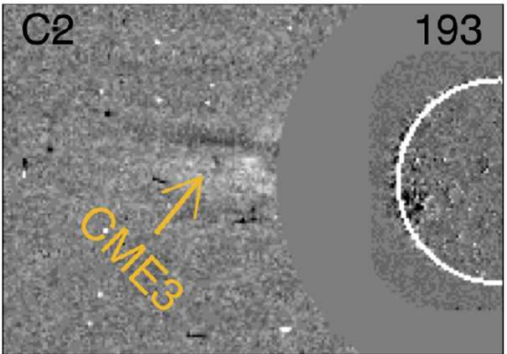

(c2) $2018101314: 36$

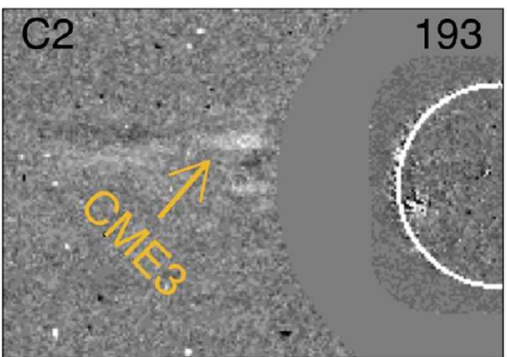

(c3) $2018101314: 48$

FIGURE 5 | The composite images of LASCO C2 WL and AIA $193 \AA$ running difference images show the propagations of the three faint CMEs. (a1-a3) for CME1, (b1-b3) for CME2, and (c1-c3) for CME3.

The results are shown in Figures 8a2,a3. In order to further calculate the heights of Shock 1 and Shock 2 from the derived local plasma densities $n$, we apply the coronal plasma density model of Sittler and Guhathakurta (1999) (see also Figure 8a1),

$$
\begin{gathered}
n(z)=n_{0} a_{1} z^{2} e^{a_{2} z}\left[1+a_{3} z+a_{4} z^{2}+a_{5} z^{3}\right] \\
z=1 /(1+y), a_{1}=0.001292, a_{2}=4.8039, \\
a_{3}=0.29696, a_{4}=-7.1743, a_{5}=12.321,
\end{gathered}
$$

where $y$ is the height above the solar surface in solar radii and $n_{0}$ is the electron number density at the solar surface. We choose $n_{0}$ as $6.0 \times 10^{8} \mathrm{~cm}^{-3}$ considering the events under this study occurring in the period of solar activity minimum, which is similar to the value used in Ma et al. (2011). The time distance profiles of Shock 1 and Shock 2 calculated from the different splitting bands in the radio dynamic spectra are presented in the panels (c1) and (c2) of Figure 8, respectively. It can be seen that the heights of Shock 1 and Shock 2 approximately change from $1.45 \mathrm{R}_{\odot}$ to 1.60 $\mathrm{R}_{\odot}$ and from $1.37 \mathrm{R}_{\odot}$ to $1.46 \mathrm{R}_{\odot}$, respectively.

The leading edges (LE1-LE3) of CME1-CME3 can be tracked in the AIA running difference images at $193 \AA$ A. For each event, we chose three different slits (s1a-s1c, s2a-s2c, and s3a-s3c in Figure 6) to make the time-distance slit images, which display the propagations of the leading edges along different directions. As examples, three time-distance diagrams from $s 1 b, s 2 b$ and $s 3 b$ are plotted in the panels (b1-b3) of Figure 8. According to the tracks or stripes in the slit images, we calculated the heights of LE1-LE3 and derived their speeds, which are shown by the diamonds in Figures 8c1-c3,d1-d3, respectively.

From Figure 8c1, it can be seen that although LE1 had moved out of the AIA's FOV when Shock1 began to appear, the development trend of the LE1's heights and the height variation of Shock1 suggest a high degree correlation between them. 


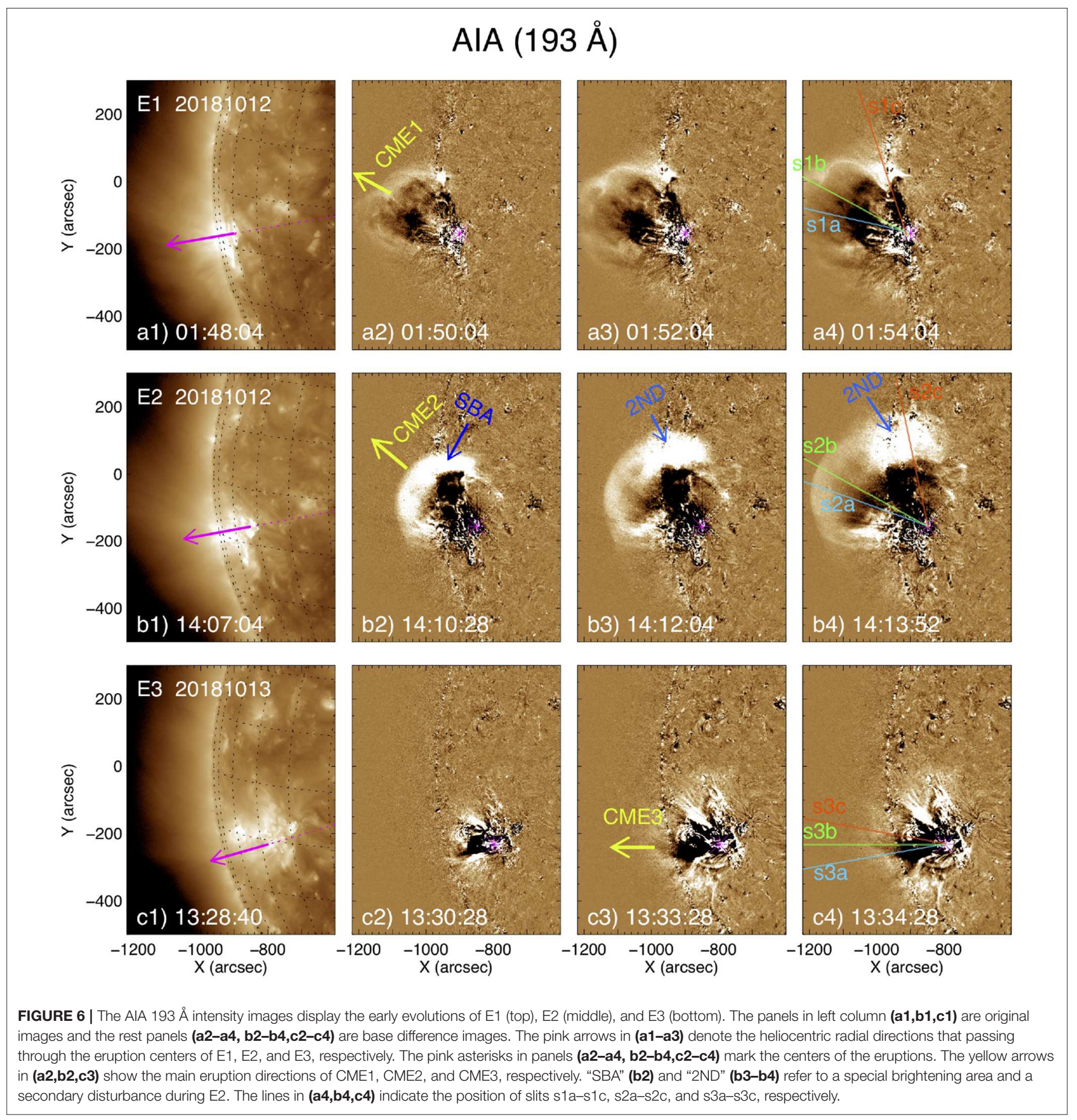

Figure 8c2 indicates a similar situation for LE2 and Shock2. Thus, it is likely that the two shocks corresponding to the two Type II radio bursts in E1 and E2 were separately triggered by the expanding of the leading edges of CME1 and CME2. In Figures 8d1,d2, it can be found that LE1 and LE2 have various speeds along different propagation directions. LE1 has the largest velocity of $\sim 320 \mathrm{~km} \mathrm{~s}^{-1}$ (azure diamonds in Figure 8d1) along s1a (azure line in Figure 6a4). The fastest speed of LE2 is $\sim 380$ $\mathrm{km} \mathrm{s}^{-1}$ (red diamonds in Figure 8d2), which was calculated along the slit s2c (red line in Figure 6b4).

The speeds of Shock 1 and Shock2 derived from their heights are given in Figures 8d1,d2, respectively. It can be found that the shocks' speeds calculated along the different Type II bursts bands are also different. These different speeds represent the speeds of the downstream (blue pluses) and upstream (red and black pluses) shock regions, which might be distinct from each other. 


\section{EUVI (195 Å)}
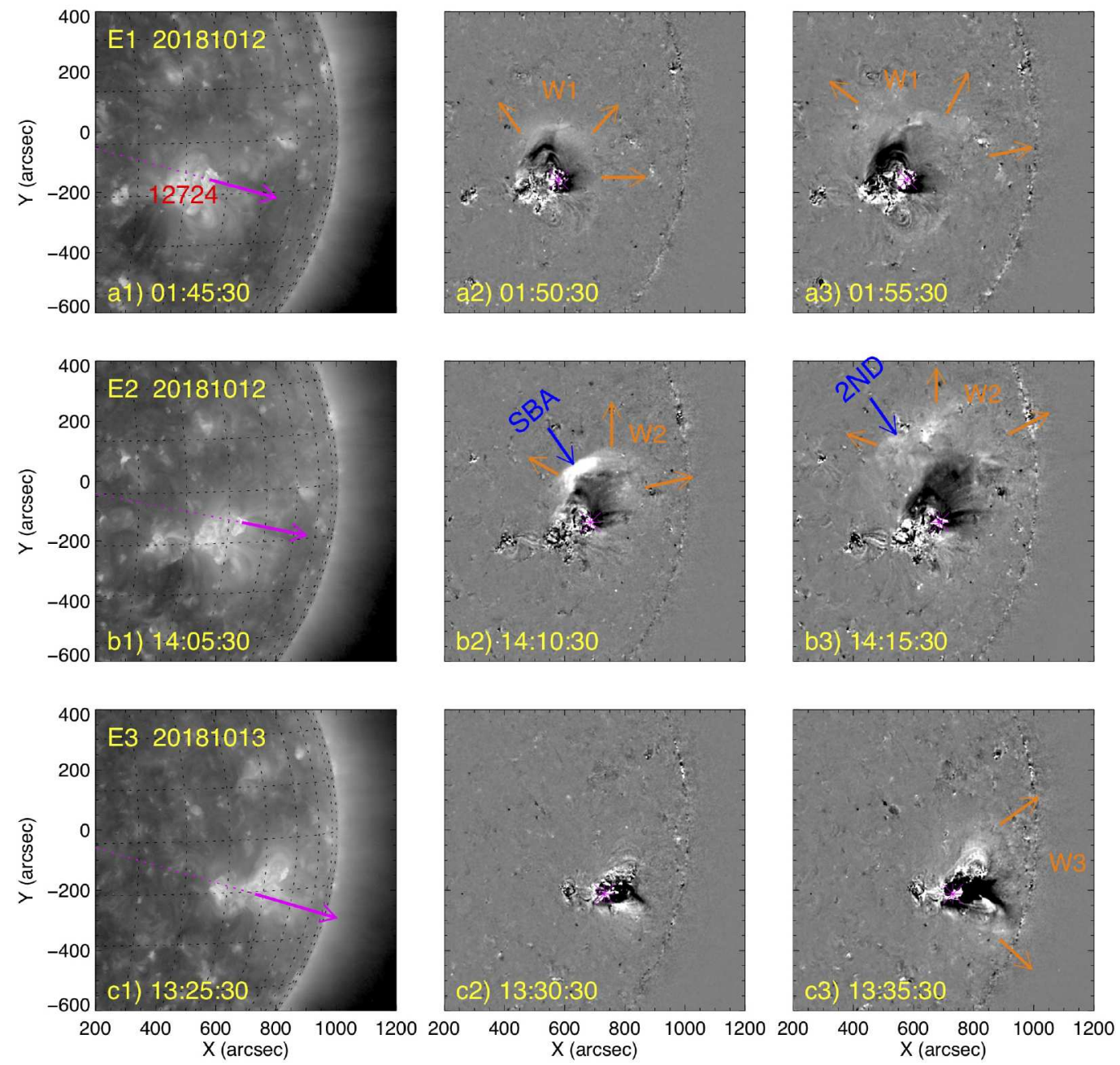

FIGURE 7 | The EUVI $195 \AA$ intensity images show the evolutions of E1 (a1-a3), E2 (b1-b3), and E3 (c1-c3) from on-disk perspective. The left column (a1,b1,c1) are the original images and the rest panels give the base difference images. Same to Figure $\mathbf{6}$, the pink arrows in the left column images (a1,b1,c1) show the heliocentric radial directions through each eruption centers (pink asterisks) The orange arrows indicate the propagation directions of the associated EUV wave "W1" (a2,a3), "W2" (b2,b3), and "W3" (c3)

In addition, the discrepancies of the speeds are also probably caused by the measurement errors. On average, Shock1 has an initial speed of $\sim 400 \mathrm{~km} \mathrm{~s}^{-1}$ and that of Shock 2 is $\sim 430 \mathrm{~km} \mathrm{~s}^{-1}$. According to the relationship between the shock's speed $\left(V_{s}\right)$ and the local Alfvén speed $V_{A}$, i.e.,

$$
M_{A}=\frac{V_{s}}{V_{A}}
$$

the Alfvén speeds at the early phases of Shock1 (01:54:32 UT, indicated by the vertical line in Figures $\mathbf{2 b}, \mathbf{8 d 1}$ ) and Shock2 (14:15:54 UT, indicated by the vertical line in Figures $\mathbf{3 b}$, 8d2) can be deduced as $\sim 300$ and $\sim 350 \mathrm{~km} \mathrm{~s}^{-1}$, respectively. Compared with the fastest speeds of LE1 $\left(\sim 320 \mathrm{~km} \mathrm{~s}^{-1}\right)$ and LE2 $\left(\sim 380 \mathrm{~km} \mathrm{~s}^{-1}\right)$, the local Alfvén speeds are smaller. These results are in agreement with the scenario of piston-driven shock, supporting our conjecture that the CMEs in E1 and E2 excited their relevant shocks and Type II radio bursts. Figure $\mathbf{8 d} \mathbf{3}$ shows that the leading edge of CME3 had a relatively slower speed $\left(\sim 160 \mathrm{~km} \mathrm{~s}^{-1}\right)$ than LE1 and LE2. It is likely less than the local Alfvén speed, which might be the reason why Type II radio burst or shock is absent in E3.

\subsection{Background Fields From PFSS Extrapolation}

To probe the background field structures surrounding the eruption source area and their relationship with the eruptions, an extrapolation was performed using the potential field source surface (PFSS) model (e.g., Schatten et al., 1969; Schrijver and De Rosa, 2003) with a starting radius of $1.01 \mathrm{R}_{\odot}$. For better reliability, one HMI longitudinal magnetogram on 2018 Oct 13 


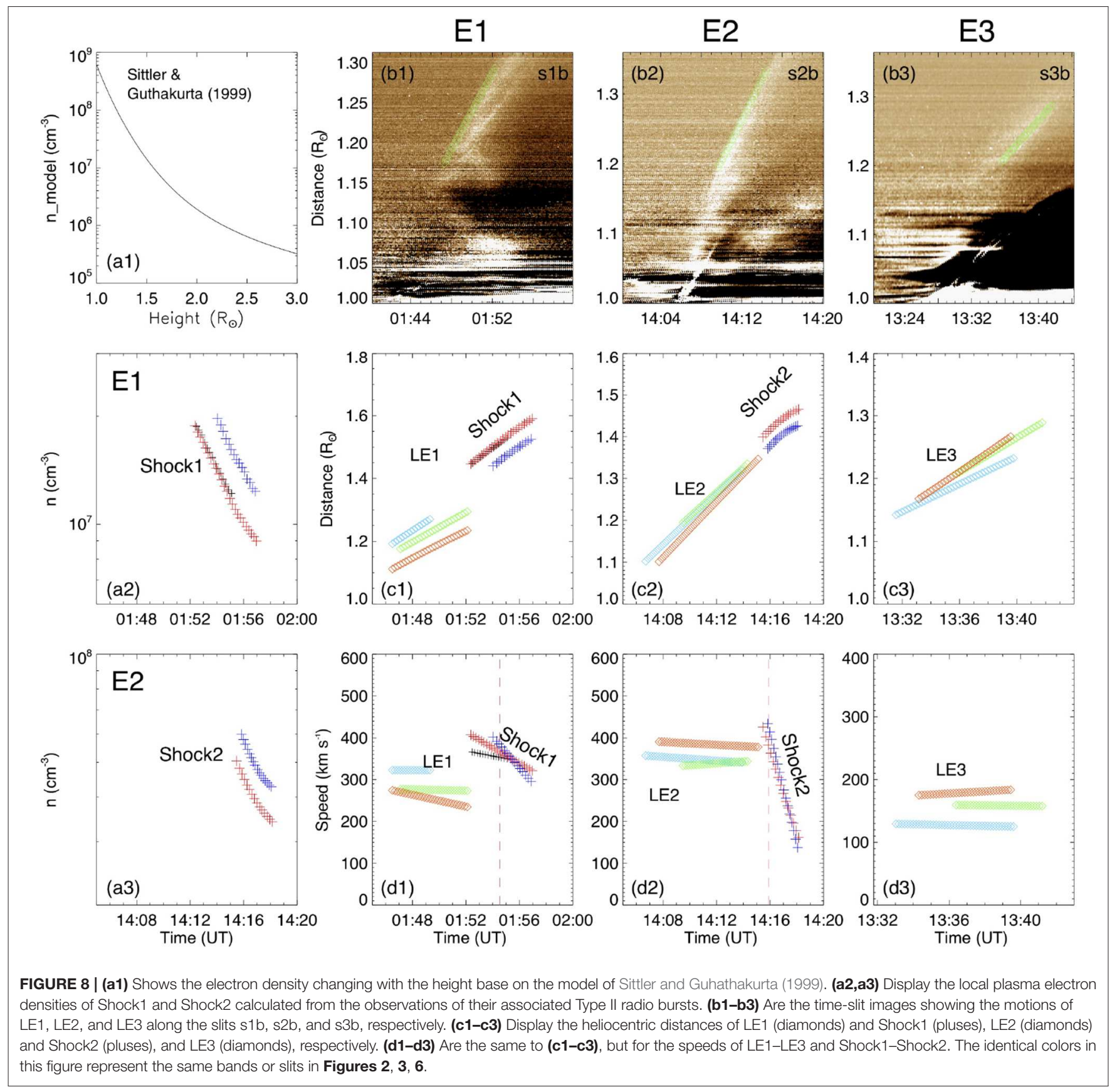

was applied to extrapolate the potential field. The magnetic field lines from the extrapolation are overlaid on the AIA $171 \AA$, EUVI $195 \AA$ intensity images, HMI and rotated HMI magnetograms, as displayed by Figures 9a-d.

The HMI magnetogram in Figure 9d shows that AR 12724 mainly consists of the leading positive flux " $\mathrm{P}$ " and following negative flux "N," with some surrounding parasitic magnetic elements, such as the fluxes "n" and "p." In this panel, we also overlaid the profiles of the three filaments F1-F3, which are indicated by the red curves. It can be seen that F1 and F2 are located at the northwest of the AR and aligned along the magnetic neutral lines between $\mathrm{N}$ and p, while F3 lies in the AR's southeast region between the opposite polarity fluxes $\mathrm{P}$ and $\mathrm{n}$. The spine directions of F1 and F2 are approximately from north to south, opposite to the east-west orientation of F3.

At the remote region to the north of AR 12724, we found some different magnetic loop systems, which are represented by the short dark blue field lines. Between these magnetic systems and the magnetic loops inside AR 12724, the magnetic quasi-separatrix layer (indicated by the yellow dashed line) may exist. That area is consistent with the place where the special brightening region SBA in E2 appeared. It is likely that SBA was 

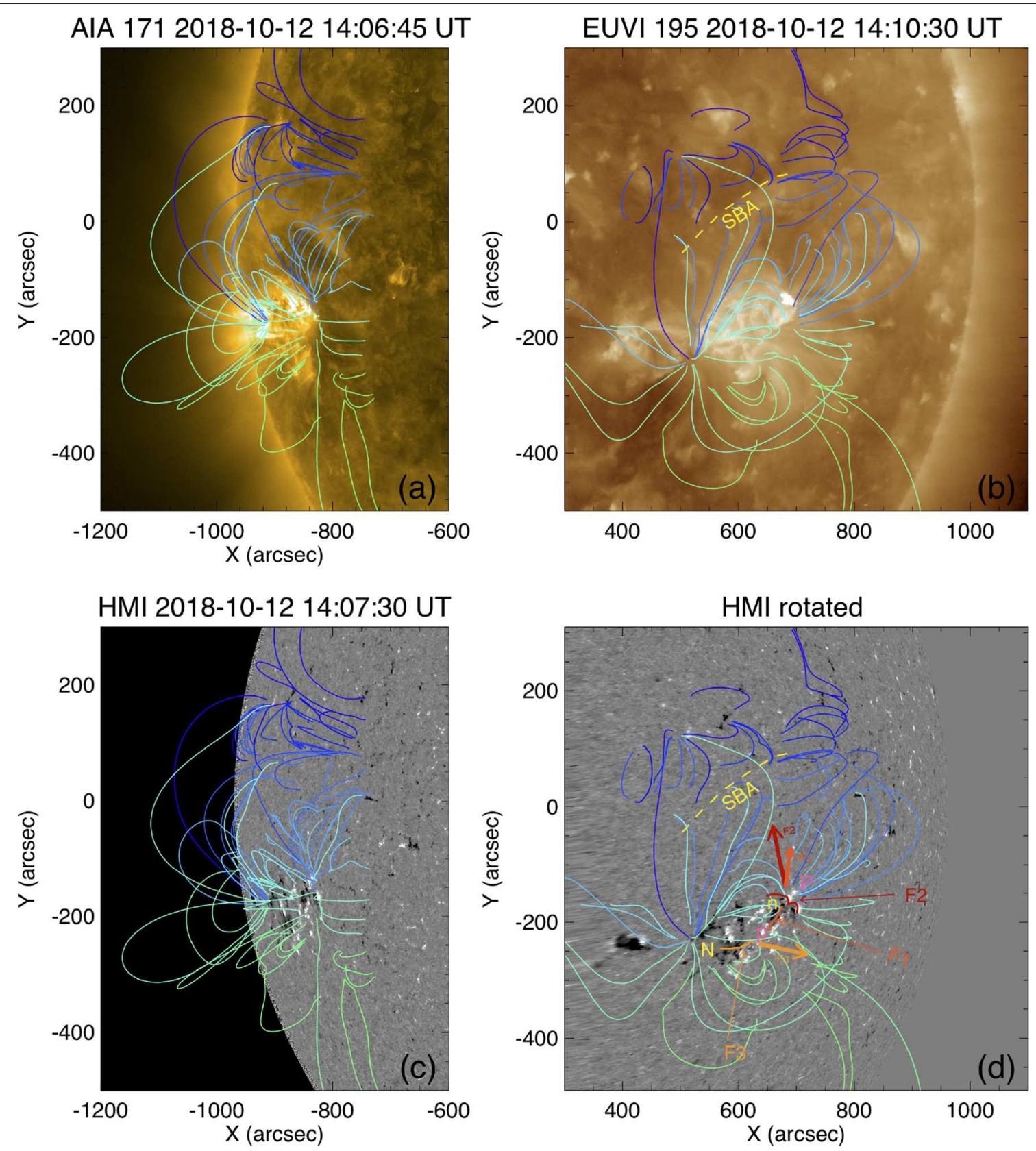

FIGURE 9 | The extrapolated magnetic field lines using the PFSS model are overlaid on the AIA intensity image (a), the EUVI intensity image (b) and HMI magnetograms (c,d). The yellow dashed curves in (b,d) indicate the location of the "SBA." "P" and "N" in (d) mark the leading and following magnetic fluxes of AR 12724. "p" and " $n$ " in (d) denote the positive and negative parasitic magnetic elements surrounding $\mathrm{N}$ and $\mathrm{P}$, respectively. The thick arrows in different colors in (d) point to the main eruption directions of F1-F3.

caused by the interaction between the north flank of CME2 and the magnetic separatrix layer.

\section{SUMMARY AND DISCUSSION}

Using radio dynamic spectra and dual perspective EUV observations, we investigate three successive solar eruptions (E1, E2, and E3) from the same active region AR 12724. All the eruptions were accompanied by a B-class flare and a poor WL CME. However, only the first two of them were observed to correlate with Type II radio bursts, suggesting the likely appearances of shocks (Shock1 and Shock2) only in the two events. From the radio dynamic spectra, we utilize the splitting bands of the Type II bursts to estimate the density jumps (1.44 and 1.30), Alfvén Mach numbers (1.35 and 1.23), and coronal Alfvén speeds $(\sim 300$ and $\sim 350 \mathrm{~km}$ $\mathrm{s}^{-1}$ ) of Shock 1 and Shock2. Through a comparative study, we found that 
- The apparent speeds of the CMEs' leading edges (LE1-LE3) are different. LE3 has an obvious slower speed $\left(\sim 160 \mathrm{~km} \mathrm{~s}^{-1}\right)$ than LE1 $\left(\sim 320 \mathrm{~km} \mathrm{~s}^{-1}\right)$ and LE2 $\left(\sim 380 \mathrm{~km} \mathrm{~s}^{-1}\right)$. The speeds of LE1 and LE2 can exceed their corresponding local Alfvén speeds $\left(\sim 300\right.$ and $\left.\sim 350 \mathrm{~km} \mathrm{~s}^{-1}\right)$.

- E1 and E2 originated from the northwest of AR 12724, while E3 took place from the AR's southeast region.

- The EUV imaging observations from two different perspective indicate that E1 and E2 erupted along the $\sim 45^{\circ}$ north of their radial directions, while CME3 in E3 approximately propagated radially.

\subsection{Trigger of Type II Radio Bursts}

According to the GOES soft X-ray flux data, Flare3 is a B2.3-class flare and stronger than the B2.1 Flare1. However, Type II radio burst is associated with the weaker one. This is in agreement with the finding that the magnitudes of flares are not directly related to the occurrence of Type II radio bursts (e.g., Cliver et al., 1999). In addition, since all the eruptions took place from the same AR and all the associated flares are relatively weak (only B-class), it is hard to conclude that the Type II bursts studied here were initiated by the blast wave due to flares. Our calculations have shown that the speeds of LE1 and LE2 along certain directions can exceed the local coronal Alfvén speeds, which meet the requirements of the formation of a piston-driven shock. Thus, It would be more reasonable that the Type II bursts were triggered by their associated CMEs. On the other, it should be noted that the third eruption E3 has a different source region and eruption direction from E1 and E2. The coronal plasma and magnetic field environments that the erupting structures of E3 encountered would be also distinct from those of E1 and E2. The missing of Type II burst in E3 may be associated with this situation as well.

\subsection{SBA and the Secondary Wave}

In the eruption E2, a special brightening area SBA is detected where a magnetic separatrix may exist according to the results of the PFSS extrapolation (Figure 9). The occurrence or appearance of SBA in this event might be explained by this scenario: when the flank of CME2 and/or EUV wave W2 arrived the magnetic separatrix layer, it would be likely compressed and heated, which might give birth to SBA. Along with the occurrence of the reflection and refraction of the EUV wave near the magnetic separatrix, a secondary wave 2ND might be further produced and propagate outward. Similar situations can be found in some other studies (e.g., Ofman and Thompson, 2002; Shen and Liu, 2012; Chandra et al., 2016; Zheng et al., 2018). In addition, some studies

\section{REFERENCES}

Benz, A. O., Monstein, C., Meyer, H., Manoharan, P. K., Ramesh, R., Altyntsev, A., et al. (2009). A world-wide net of solar radio spectrometers: eCALLISTO. Earth Moon Planets 104, 277-285. doi: 10.1007/s11038-0089267-6

Brueckner, G. E., Howard, R. A., Koomen, M. J., Korendyke, C. M., Michels, D. J., Moses, J. D., et al. (1995). The large angle spectroscopic coronagraph (LASCO). Solar Physics, 162:357. have shown a close relationship between Type II radio bursts and such interactions (e.g., Feng et al., 2012; Kong et al., 2012; Shen et al., 2019). Using simultaneous radio and EUV imaging data, Chen et al. (2014) found that the source location of a solar type II radio burst coincides with the interface between CME EUV wave front and a nearby coronal ray structure, where an obvious EUV brightening also appeared. They conjectured that the CME streamer interactions may be important to the formation of type II radio burst. Unfortunately, there is no radioheliograph observation available for our study and we can not confirm the exact location of the type II burst in E2, but according to the results of Chen et al. (2014), it can be suspected that the special brightening area SBA corresponds to the source region of the type II burst in E2.

\section{DATA AVAILABILITY STATEMENT}

Publicly available datasets were analyzed in this study. This data can be found here: http://sdac.virtualsolar.org/cgi/search, https://rsdb.obs-nancay.fr, http://soleil.i4ds.ch/solarradio/callisto Quicklooks/.

\section{AUTHOR CONTRIBUTIONS}

SM downloaded and analyzed the data in both radio and EUV passband, and wrote the paper. HC analyzed the data in EUV passband and wrote the paper.

\section{FUNDING}

This work was supported by NSFC (11433006, 11533008, $11661161015,11790300,11790301,11790304,11941003$, 11973057, and 41331068) and the B-type Strategic Priority Program of the Chinese Academy of Sciences, Grant No. XDB41000000.

\section{ACKNOWLEDGMENTS}

We were grateful to Prof. Jun Lin and Prof. Baolin Tan for their insightful suggestions and informative discussions. We acknowledge NASA's open data policy in using SDO data. STEREO is a mission in NASA's Solar Terrestrial Probes program. $\mathrm{SOHO}$ is a project of international collaboration between ESA and NASA. We were grateful to ORFEES, Learmonth and Greenland teams for offering the radio spectra data. We thank the referees for their constructive comments and suggestions. 
Cliver, E. W., Webb, D. F., and Howard, R. A. (1999). On the origin of solar metric type II bursts. Sol. Phys. 187, 89-114. doi: 10.1023/A:1005115119661

Dauphin, C., Vilmer, N., and Krucker, S. (2006). Observations of a soft Xray rising loop associated with a type II burst and a coronal mass ejection in the 03 November 2003 X-ray flare. Astron. Astrophys. 455, 339-348. doi: 10.1051/0004-6361:20054535

Dodge, J. C. (1975). Source regions for type II radio bursts. Sol. Phys. 42, 445-459. doi: 10.1007/BF00149925

Dodson, H. W., Hedeman, E. R., and Owren, L. (1953). Solar flares and associated $200 \mathrm{Mc} / \mathrm{sec}$ radiation. Astrophys. J. 118:169. doi: 10.1086/145740

Eselevich, V. G., Eselevich, M. V., and Zimovets, I. V. (2019). Observations of a flare-generated blast wave in a pseudo coronal mass ejection event. Sol. Phys. 294:73. doi: 10.1007/s11207-019-1467-x

Eselevich, V. G., Eselevich, M. V., Zimovets, I. V., and Sharykin, I. N. (2017). Evidence for shock generation in the solar corona in the absence of coronal mass ejections. Astron. Rep. 61, 805-819. doi: 10.1134/S1063772917080030

Feng, S. W., Chen, Y., Kong, X. L., Li, G., Song, H. Q., Feng, X. S., et al. (2012). Radio signatures of coronal-mass-ejection-streamer interaction and source diagnostics of type II radio burst. Astrophys. J. 753:21. doi: $10.1088 / 0004-637 X / 753 / 1 / 21$

Frassati, F., Susino, R., Mancuso, S., and Bemporad, A. (2019). Comprehensive analysis of the formation of a shock wave associated with a coronal mass ejection. Astrophys. J. 871:212. doi: 10.3847/1538-4357/aaf9af

Giovanelli, R. G., and Roberts, J. A. (1958). Optical observations of the solar disturbances causing type II radio bursts. Aust. J. Phys. 11:353. doi: 10.1071/PH580353

Gopalswamy, N., Lara, A., Kaiser, M. L., and Bougeret, J. L. (2001). Near-sun and near-earth manifestations of solar eruptions. J. Geophys. Res. 106, 25261-25278. doi: 10.1029/2000JA004025

Gosling, J. T., Hildner, E., MacQueen, R. M., Munro, R. H., Poland, A. I., and Ross, C. L. (1976). The speeds of coronal mass ejection events. Sol. Phys. 48, 389-397. doi: 10.1007/BF00152004

Grechnev, V. V., Lesovoi, S. V., Kochanov, A. A., Uralov, A. M., Altyntsev, A. T., Gubin, A. V., et al. (2018). Multi-instrument view on solar eruptive events observed with the Siberian Radioheliograph: from detection of small jets up to development of a shock wave and CME. J. Atmos. Solar Terres. Phys. 174, 46-65. doi: 10.1016/j.jastp.2018.04.014

Howard, R. A., Moses, J. D., Vourlidas, A., Newmark, J. S., Socker, D. G., Plunkett, S. P., et al. (2008). Sun earth connection coronal and heliospheric investigation (SECCHI). Space Sci. Rev. 136, 67-115. doi: 10.1007/s11214-008-9341-4

Huang, J., Démoulin, P., Pick, M., Auchére, F., Yan, Y. H., and Bouteille, A. (2011). Initiation and early development of the 2008 April 26 coronal mass ejection. Astrophys. J. 729:107. doi: 10.1088/0004-637X/729/2/107

Kaiser, M. L., Kucera, T. A., Davila, J. M., St. Cyr, O. C., Guhathakurta, M., and Christian, E. (2008). The STEREO mission: an introduction. Space Sci. Rev. 136, 5-16. doi: 10.1007/s11214-007-9277-0

Kennewell, J., and Steward, G. (2003). Solar Radio Spectrograph [SRS] Data. Sydney, NSW: IPS Radio and Space Serv.

Klassen, A., Pohjolainen, S., and Klein, K. L. (2003). Type II radio precursor and x-ray flare emission. Sol. Phys. 218, 197-210. doi: 10.1023/B:SOLA.0000013034.61996.c4

Klein, K.-L., Khan, J. I., Vilmer, N., Delouis, J.-M., and Aurass, H. (1999). X-ray and radio evidence on the origin of a coronal shock wave. Astron. Astrophys. 346, L53-L56.

Kong, X. L., Chen, Y., Li, G., Feng, S. W., Song, H. Q., Guo, F., et al. (2012). A broken solar type II radio burst induced by a coronal shock propagating across the streamer boundary. Astrophys. J. 750:158. doi: $10.1088 / 0004-637 \mathrm{X} / 750 / 2 / 158$

Lemen, J. R., Title, A. M., Akin, D. J., Boerner, P. F., Chou, C., Drake, J. F., et al. (2012). The atmospheric imaging assembly (AIA) on the solar dynamics observatory (SDO). Sol. Phys. 275, 17-40. doi: 10.1007/s11207-0119776-8

Liu, W., and Ofman, L. (2014). Advances in observing various coronal EUV waves in the SDO era and their seismological applications. Sol. Phys. 289, 3233-3277. doi: 10.1007/s11207-014-0528-4

Long, D. M., Bloomfield, D. S., Chen, P. F., Downs, C., Gallagher, P. T., Kwon, R. Y., et al. (2017). Understanding the physical nature of coronal "EIT waves". Sol. Phys. 292:7. doi: 10.1007/s11207-016-1030-y
Ma, S., Raymond, J. C., Golub, L., Lin, J., Chen, H., Grigis, P., et al. (2011). Observations and interpretation of a low coronal shock wave observed in the EUV by the SDO/AIA. Astrophys. J. 738:160. doi: $10.1088 / 0004-637 X / 738 / 2 / 160$

Ma, S., Wills-Davey, M. J., Lin, J., Chen, P. F., Attrill, G. D. R., Chen, H., et al. (2009). A new view of coronal waves from STEREO. Astrophys. J. 707, 503-509. doi: $10.1088 / 0004-637 X / 707 / 1 / 503$

Mann, G., Classen, T., and Aurass, H. (1995). Characteristics of coronal shock waves and solar type II radio bursts. Astron. Astrophys. 295:775.

Maxwell, A., and Thompson, A. R. (1962). Spectral observations of solar radio bursts. II. Slow-drift bursts and coronal streamers. Astrophys. J. 135:138. doi: $10.1086 / 147254$

Nelson, G. J., and Melrose, D. B. (1985). "Type II bursts," in Solar Radiophysics: Studies of Emission From the Sun at Metre Wavelengths (A87-13851 03-92), eds D. J. McLean and N. R. Labrum (Cambridge; New York, NY: Cambridge University Press), 333-359.

Nindos, A., Alissandrakis, C. E., Hillaris, A., and Preka-Papadema, P. (2011). On the relationship of shock waves to flares and coronal mass ejections. Astron. Astrophys. 531:A31. doi: 10.1051/0004-6361/201116799

Nindos, A., Aurass, H., Klein, K. L., and Trottet, G. (2008). Radio emission of flares and coronal mass ejections. Sol. Phys. 253, 3-41. doi: 10.1007/s11207-008-9258-9

Ning, Z. (2016). Bi-directional flows in a C-class solar flare. Astrophys. Space Sci. 361:22. doi: 10.1007/s10509-015-2606-y

Ofman, L., and Thompson, B. J. (2002). Interaction of EIT waves with coronal active regions. Astrophys. J. 574, 440-452. doi: 10.1086/340924

Payne-Scott, R., Yabsley, D. E., and Bolton, J. G. (1947). Relative times of arrival of bursts of solar noise on different radio frequencies. Nature 160, 256-257. doi: 10.1038/160256b0

Pesnell, W. D., Thompson, B. J., and Chamberlin, P. C. (2012). The solar dynamics observatory (SDO). Sol. Phys. 275, 3-15. doi: 10.1007/s11207-011-9841-3

Reid, H. A. S., and Ratcliffe, H. (2014). A review of solar type III radio bursts. Res. Astron. Astrophys. 14, 773-804. doi: 10.1088/1674-4527/14/7/003

Schatten, K. H., Wilcox, J. M., and Ness, N. F. (1969). A model of interplanetary and coronal magnetic fields. Sol. Phys. 6, 442-455. doi: 10.1007/BF00146478

Schrijver, C. J., and De Rosa, M. L. (2003). Photospheric and heliospheric magnetic fields. Sol. Phys. 212, 165-200. doi: 10.1023/A:1022908504100

Shen, Y., Chen, P. F., Liu, Y. D., Shibata, K., Tang, Z., and Liu, Y. (2019). First unambiguous imaging of large-scale quasi-periodic extreme-ultraviolet wave or shock. Astrophys. J. 873:22. doi: 10.3847/1538-4357/ab01dd

Shen, Y., and Liu, Y. (2012). Simultaneous observations of a large-scale wave event in the solar atmosphere: from photosphere to corona. Astrophys. J. Lett. 752:L23. doi: 10.1088/2041-8205/752/2/L23

Sittler, E. C. J.r., and Guhathakurta, M. (1999). Semiempirical two-dimensional magnetohydrodynamic model of the solar corona and interplanetary medium. Astrophys. J. 523, 812-826. doi: 10.1086/307742

Smerd, S. F., and Dulk, G. A. (1971). In Solar Magnetic Fields, Vol. 43 of IAU Symposium, ed R. Howard (Dordrecht: Reidel), 616. doi: $10.1017 /$ S0074180900023081

Smerd, S. F., Sheridan, K. V., and Stewart, R. T. (1974). "On split-band structure in type II radio bursts from the sun," in Coronal Disturbances, Vol. 57 of IAU Symposium, ed G. A. Newkirk (Dordrecht; Boston: Reidel), 389. doi: $10.1017 /$ S0074180900234542

Stewart, R. T. (1985). Moving Type IV Bursts. Cambridge, New York, NY: Cambridge University Press. 361-383.

Su, W., Cheng, X., Ding, M. D., Chen, P. F., and Sun, J. Q. (2015). A type II radio burst without a coronal mass ejection. Astrophys. J. 804:88. doi: 10.1088/0004-637X/804/2/88

Swarup, G., Stone, P. H., and Maxwell, A. (1960). The association of solar radio bursts with flares and prominences. Astrophys. J. 131:725. doi: 10.1086/146885

Tan, B., Mészárosová, H., Karlický, M., Huang, G., and Tan, C. (2016) Microwave type III pair bursts in solar flares. Astrophys. J. 819:42. doi: 10.3847/0004-637X/819/1/42

Vlahos, L., Gergely, T. E., and Papadopoulos, K. (1982). Electron acceleration and radiation signatures in loop coronal transients. Astrophys. J. 258, 812-822. doi: $10.1086 / 160128$

Vršnak, B. (2001). Solar flares and coronal shock waves. J. Geophys. Res. 106, 25291-25300. doi: 10.1029/2000JA004009 
Vršnak, B., and Cliver, E. W. (2008). Origin of coronal shock waves. Sol. Phys. 253, 215-235. doi: 10.1007/s11207-008-9241-5

Vršnak, B., Magdalenić, J., Aurass, H., and Mann, G. (2002). Band-splitting of coronal and interplanetary type II bursts. II. Coronal magnetic field and Alfvén velocity. Astron. Astrophys. 396, 673-682. doi: 10.1051/0004-6361:20021413

Warmuth, A. (2015). Large-scale globally propagating coronal waves. Living Rev. Sol. Phys. 12:3. doi: 10.1007/lrsp-2015-3

Wild, J. P., and McCready, L. L. (1950). Observations of the spectrum of high-intensity solar radiation at metre wavelengths. I. The apparatus and spectral types of solar burst observed. Aust. J. Sci. Res. A Phys. Sci. 3:387. doi: 10.1071/CH9500387

Wild, J. P., Murray, J. D., and Rowe, W. C. (1954). Harmonics in the spectra of solar radio disturbances. Aust. J. Phys. 7:439. doi: 10.1071/PH540439

Wuelser, J.-P., Lemen, J. R., Tarbell, T. D., Wolfson, C. J., Cannon, J. C., Carpenter, B. A., et al. (2004). "EUVI: the STEREO-SECCHI extreme ultraviolet imager," in Proceedings of the SPIE, Vol. 5171 of Society of Photo-Optical Instrumentation Engineers (SPIE) Conference Series, eds S. Fineschi and M. A. Gummin (San Diego, CA), 111-122. doi: 10.1117/12.506877
Yan, Y., Pick, M., Wang, M., Krucker, S., and Vourlidas, A. (2006). A radio burst and its associated CME on March 17, 2002. Sol. Phys. 239, 277-292. doi: 10.1007/s11207-0060202-6

Zheng, R., Chen, Y., Feng, S., Wang, B., and Song, H. (2018). An extremeultraviolet wave generating upward secondary waves in a streamer-like solar structure. Astrophys. J. Lett. 858:L1. doi: 10.3847/2041-8213/aabe87

Conflict of Interest: The authors declare that the research was conducted in the absence of any commercial or financial relationships that could be construed as a potential conflict of interest.

Copyright (C) $2020 \mathrm{Ma}$ and Chen. This is an open-access article distributed under the terms of the Creative Commons Attribution License (CC BY). The use, distribution or reproduction in other forums is permitted, provided the original author(s) and the copyright owner(s) are credited and that the original publication in this journal is cited, in accordance with accepted academic practice. No use, distribution or reproduction is permitted which does not comply with these terms. 